\title{
The quantity of unheated milk tolerated as a predictor of tolerance to baked milk
}

\author{
Sait Karaman, Semiha Bahçeci Erdem, Hikmet Tekin Nacaroğlu, Canan Şule Karkıner, Demet Can
}

\begin{abstract}
Background: Tolerance of baked milk indicates a good prognosis in IgE-mediated cow's milk allergy.

Objective: The present study aims to investigate the predictors of baked milk tolerance, particularly the amount of milk tolerated in the first oral food challenge (OFC) test, in children with IgE-mediated cow's milk allergy.

Methods: The study included 35 cases who were diagnosed with IgE-mediated cow's milk allergy upon open OFC testing in the Pediatric Allergy Clinic. Four weeks after the diagnosis, skin prick test (SPT) and OFC were performed with baked milk. Cases who did and did not develop reactions during OFC with baked milk were compared regarding clinical and laboratory parameters.

Results: Twelve cases (33.3\%) did not develop a reaction during OFC with baked milk. Those who had low levels of casein sIgE, $\beta$-lactoglobulin sIgE, and $\alpha$-lactoalbumin sIgE; small SPT wheal diameter for baked milk and a-lactoalbumin; and a large amount of unheated milk tolerated in the first OFC were found to be tolerant to baked milk ( $\mathrm{p}<0.05)$. For predicting baked milk tolerance, a cut-off level of the amount of unheated milk tolerated in OFC was calculated as $620 \mathrm{mg}$ [with the area under the curve (AUC) 0.88 (95\% confidence interval 0.77-0.99) in ROC curve analysis].
\end{abstract}

Conclusion: If a child with cow's milk allergy is able to tolerate more than $620 \mathrm{mg}$ of milk protein during challenge with unheated milk, this may show that this child will tolerate baked milk, meaning that the child will be able to tolerate cow's milk in the future.

Key words: Allergy, Baked, Casein, Challenge, Children, Food, Milk, Predictor, Tolerance

\section{Citation:}

Karaman, S., Erdem, S. B., Nacaroğlu, H. T., Karkıner, C. Ş., Can, D. (2022). The quantity of unheated milk tolerated as a predictor of tolerance to baked milk.

Asian Pac J Allergy Immunol, 40(4), 353-358.

https://doi.org/10.12932/ap-280419-0549

\section{Affiliation:}

Department of Pediatric Allergy, Dr Behcet Uz Children's Hospital, Izmir, Turkey

Corresponding author:

Sait Karaman

Dr Behcet Uz Children's Hospital, Department of Pediatric Allergy and Immunology, Izmir, Turkey

E-mail: saitkaraman73@gmail.com

\section{Introduction}

Cow's milk allergy is one of the most common childhood food allergies, with a prevalence of $2-3 \%$ in children. ${ }^{1}$ Today, the treatment of patients with cow's milk allergy consists of the elimination of milk and dairy products from the diet. However, since many foods contain milk proteins, it is very difficult to prevent exposure to milk by the elimination of dairy products from diet alone. ${ }^{2}$ In recent years, evidence has shown that $65-83 \%$ of patients allergic to milk can eat baked milk products without any reaction. ${ }^{3,4}$ Regular ingestion of baked milk products may accelerate acquisition of tolerance to cow's milk. ${ }^{5}$ Furthermore, milkallergic patients who tolerate baked milk are unlikely to present with a severe reaction when exposed to unheated milk. ${ }^{3}$ Another option in the treatment of milk allergy is desensitization. Studies show that instead of desensitization with unheated milk, baked milk may be a better option due to its convenience and fewer side effects. ${ }^{5-7}$ In order to determine whether or not a patient with milk allergy can tolerate baked 
milk products, it is recommended to perform an oral food challenge with baked milk. Since OFC with baked milk is a time consuming test, there is a need for a marker that can predict tolerance to baked milk without OFC. Many studies have shown that the larger the SPT wheal and the higher the specific IgE level to cow's milk, the less likely the patient will tolerate baked milk..$^{4,8,9}$ In addition to this, diagnostic tests that use baked milk products are better at reflecting reactivity against baked milk. ${ }^{10}$

In this study, we investigated possible markers that could predict tolerance to baked milk products in children with IgE-mediated cow's milk allergy that was confirmed with OFC.

\section{Materials and Methods \\ Study design}

This prospective study was conducted in the Department of Paediatric Allergy in Dr. Behçet Uz Children's Hospital from January 2015 to January 2016. The study was approved by the local Research Ethics Board of Dr. Behçet Uz Children's Hospital (protocol number 2014/16, decision approval 2014/ 02-05). All cases with a history of suspicion for IgE-mediated cow's milk allergy were screened. All children or their guardians signed an informed consent form after they were notified about the study, including the possibility of severe reactions. All children had SPT to unheated milk, casein, a-lactoalbumin, and $\beta$-lactoglobulin documented in the medical record prior to oral food challenge. In all children,
ssIgE for milk, casein, $\alpha$-lactoalbumin, and $\beta$-lactoglobulin were measured prior to oral food challenge. Serum samples were analyzed for ssIgE using an UniCAP 100 system fluorescence enzyme immunoassay (Phadia, Uppsala, Sweden). The lowest limit of detection of the assay was $0.35 \mathrm{kU}_{\mathrm{A}} / \mathrm{L}$. Cases with suspected milk allergy were subjected to OFC test following the elimination of milk and dairy products from the diet for 4 weeks. Cases with milk allergy confirmed by OFC test were first subjected to SPT with baked milk and subsequently to OFC with baked milk. One case was excluded from the study because of anaphylaxis developed during OFC with unheated milk (Figure 1).

\section{Patients}

The study was conducted on cases with IgE-mediated cow's milk allergy who met the following criteria:

1. History of suspicion for IgE-mediated cow's milk allergy, and

2. Skin prick test (SPT) wheal diameter and serum specific IgE (ssIgE) levels were below the cut-off point (milk-specific IgE levels $\leq 5 \mathrm{kU}_{\mathrm{A}} / \mathrm{L}$ and SPT wheal diameter $\leq 6 \mathrm{~mm}),{ }^{11}$ and

3. Diagnosis of cow's milk allergy was confirmed with positive cow's milk oral challenge performed after four weeks of abstinence from milk and dairy products. ${ }^{11,12}$ and

4. To be older than six months old.

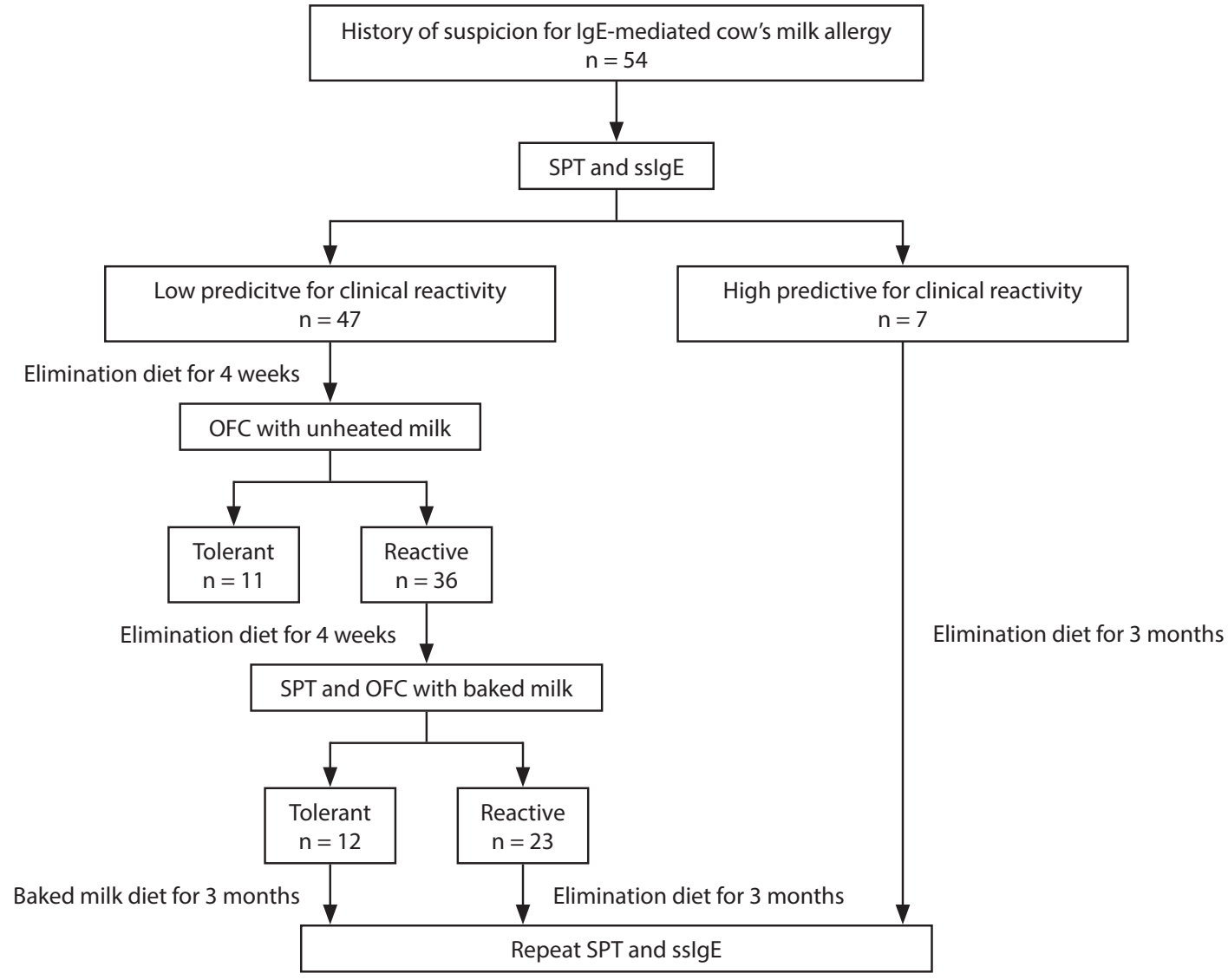

Figure 1. Study design. 

OFC.

The study exclusion criteria: History of anaphylaxis during

\section{Skin prick test}

The SPTs were performed by trained physicians on the volar surface of the forearm of each patient using a commercial extract of the milk (ALK Abelló, Horsholm, Denmark), and a prick-by-prick technique with cow milk was used. Additionally, a commercial extract of milk components (Lofarma, Milano, Italy) was also used. The tests were always performed with a histamine positive control and a saline negative control. A mean wheal diameter greater than $3 \mathrm{~mm}$ after 15 minutes of testing the allergen extracts was accepted as a positive result. During the evaluation of the SPTs for milk allergy diagnosis, the SPT wheal sizes for which the commercial extract was used were used. The diameter of the wheal determined by prick-by-prick was used while compared the diameters of SPT induration between unheated milk and baked milk.

\section{Oral food challenge}

OFCs were performed under medical supervision with emergency support available. Patients were examined and measured blood pressured prior to starting feeding, as well as before each dose was administered, ${ }^{13}$ and their vital signs and observations for lungs and skin were recorded on clinical charts. Any signs or symptoms that occurred during the OFC were also recorded, even when mild. Clinical observations continued for at least 4 hours after the last food dose. The initial dose for the OFC was $3 \mathrm{mg}$, as recommended by the European Academy of Allergy and Clinical Immunology food allergy and anaphylaxis guidelines, and a total of nine doses were given to patients that showed no reactions to the logarithmic dose increments. The maximum dose included $3 \mathrm{~g}$ of the protein. A total of 4.5 gr milk protein was applied. ${ }^{14}$ The cow's milk challenges were conducted using cow's unheated milk (or formula milk for infants less than 12 months). In OFC test, development of any symptom related to skin (itching, urticaria, angioedema), gastrointestinal system (diarrhea, vomiting, irritability), respiratory system (rhinitis, cough, wheezing, stridor, shortness of breath), or cardiovascular system (tachycardia, hypotonia, hypotension, fatigue, shock) was accepted as a positive test, and the testing was terminated.

\section{Baked milk}

Baked milk was prepared by the researchers in accordance with the literature, by cooking fresh milk for at least 30 minutes in an oven heated to $350^{\circ} \mathrm{F}^{3}$ Since $30 \%$ of the milk is vaporized during baking, the resulting baked milk was assumed to contain $5 \mathrm{gr}$ of milk protein per $100 \mathrm{ml}$. OFC with baked milk was applied as recommended by the European Academy of Allergy and Clinical Immunology food allergy and anaphylaxis guidelines. The OFC was stopped and considered positive when objective signs and symptoms (urticaria, nasal discharge, wheeze, vomiting, hypotension) or repeated severe subjective symptoms (discomfort, nausea, stomachache) were noted.
The following parameters were compared between patients who were reactive and non-reactive during OFC with baked milk:

1. The age at which the first reaction following ingestion of milk occurred

2. Gender

3. Accompanying food allergies

4. Patient's reactivity (e.g. urticaria, nasal discharge, wheeze, shortness of breath, vomiting) following the mother's consumption of milk or dairy products

5. Total eosinophil count, total IgE, ssIgE levels (for cow's milk, $\beta$-lactoglobulin, casein, $\alpha$-lactoalbumin)

6. Prick-to-prick diameter (unheated milk, baked milk)

7. SPT wheal diameter (for unheated milk, baked milk, $\beta$-lactoglobulin, casein, $\alpha$-lactoalbumin)

8. The amount of milk ingested before the development of reaction during OFC with unheated milk

\section{Statistical analysis}

IBM SPSS version 22.0 (Armonk, New York, United States) was used for all statistical analyses. The KolmogorovSmirnov test was used to test the normality of variables. Parametric methods were used for analysis of variables with a normal distribution, whereas non-parametric methods were used for analysis of variables that were not normally distributed. Comparisons of continuous variables were made with independent-samples t-test and Mann-Whitney $U$ test as appropriate. Pearson's chi-square and linear-by-linear association tests were used with an exact test for the comparison of categorical data. The categorical data are expressed as a percentage of the number (n) of children evaluated. Sensitivity and specificity between the classifications were determined using cut-off values calculated from the group variables and analyzed using a receiver operating curve (ROC). The level of significance for the analyses was $p<0.05$.

\section{Results}

In a screening of 54 cases, 36 cases met the inclusion criteria. Among these 36 cases, 1 case was excluded because of anaphylaxis. Of the 35 children included in the study, $23(65.7 \%)$ were male. The median age was 17 (7-48) months. In $14(40 \%)$ cases, milk allergy was accompanied by egg allergy. Eleven (31.4\%) of the patients on breastmilk showed reactions whenever the mother consumed milk or dairy products. In the OFC with unheated milk, the ingestion of an average of 780 (3-2850) mg of milk protein provoked the development of a reaction. Twelve $(34.3 \%)$ cases did not show reaction during OFC with baked milk. Fourteen cases had negative SPT $(<3 \mathrm{~mm})$ results for baked milk, and eight of these $(57 \%)$ could tolerate baked milk. Those who did and did not show reaction during OFC with baked milk were compared with each other. In the non-reactive group, casein-specific IgE, $\beta$-lactoglobulin-specific $\operatorname{IgE}$, a-lactoalbumin-specific IgE levels, and SPT wheal diameters for baked milk and for a-lactoalbumin were significantly lower compared to those who could not tolerate baked milk $(p<0.05)$. Additionally, the amount of milk ingested 
Table 1. Clinical and demographic properties of cases according to reactivity during OFC with baked milk.

\begin{tabular}{|c|c|c|c|}
\hline & $\begin{array}{l}\text { Reactive group } \\
\qquad(\mathbf{n}=23)\end{array}$ & $\begin{array}{l}\text { Non-reactive group } \\
\qquad(\mathrm{n}=12)\end{array}$ & $p$ value \\
\hline Gender (male), n (\%) & $15(65)$ & $8(66)$ & 1 \\
\hline Age of onset of cow's milk allergy (month)* & $5.4(4-7)$ & $6.4(3-11)$ & 0.098 \\
\hline Accompanying food allergies, $\mathrm{n}(\%)$ & $9(39)$ & $5(42)$ & 1 \\
\hline Reaction with ingestion of breastmilk, n (\%) & $8(35)$ & $3(25)$ & 0.709 \\
\hline SPT wheal diameter for unheated milk $(\mathrm{mm})^{*}$ & $5.0(1.4-7)$ & $4.5(3-7)$ & 0.380 \\
\hline SPT wheal diameter for baked milk $(\mathrm{mm})^{*}$ & $3.2(1-7)$ & $1.4(1-6)$ & $0.013^{* *}$ \\
\hline SPT wheal diameter for $\beta$-lactoglobulin $(\mathrm{mm})^{*}$ & $4.1(1-7)$ & $3.4(1-7)$ & 0.437 \\
\hline SPT wheal diameter for casein $(\mathrm{mm})^{*}$ & $3.2(1-6)$ & $1.8(1-5)$ & 0.130 \\
\hline SPT wheal diameter for $\alpha$-lactoalbumin $(\mathrm{mm})^{*}$ & $4.7(1-9)$ & $3.0(1-7)$ & $0.045^{\star *}$ \\
\hline Milk-specific $\operatorname{IgE}\left(\mathrm{kU}_{\mathrm{A}} / \mathrm{L}\right)^{*}$ & $4.5(0.3-21)$ & $2.6(0.2-11.3)$ & 0.136 \\
\hline$\beta$-lactoglobulin-specific $\operatorname{IgE}\left(\mathrm{kU}_{\mathrm{A}} / \mathrm{L}\right)^{*}$ & $18(0.4-100)$ & $1.4(0.1-3.4)$ & $0.007^{\star *}$ \\
\hline Casein-specific $\operatorname{IgE}\left(\mathrm{kU}_{\mathrm{A}} / \mathrm{L}\right)^{*}$ & $18.7(0.3-100)$ & $0.95(0.1-8.6)$ & $0.009^{* *}$ \\
\hline a-lactoalbumin-specific $\operatorname{IgE}\left(\mathrm{kU}_{\mathrm{A}} / \mathrm{L}\right)^{*}$ & $13(0.1-72)$ & $2.6(0.1-10.2)$ & $0.046^{* *}$ \\
\hline Total $\operatorname{IgE}\left(\mathrm{kU}_{\mathrm{A}} / \mathrm{L}\right)^{*}$ & $170(7-511)$ & $118(11-653)$ & 0.152 \\
\hline Total eosinophil count, $\left(\times 10^{-3} \mu \mathrm{L}\right)^{\star}$ & $425(30-830)$ & $471(40-850)$ & 0.429 \\
\hline $\begin{array}{l}\text { The amount of unheated milk ingested during } \\
\text { OFC until the development of reaction }(\mathrm{mg})^{*}\end{array}$ & $326(3-1450)$ & $1344(440-2850)$ & $0.002^{* *}$ \\
\hline
\end{tabular}

${ }^{*}$ median $(\min -\max ),{ }^{*}$ Significant $\mathrm{p}$ value

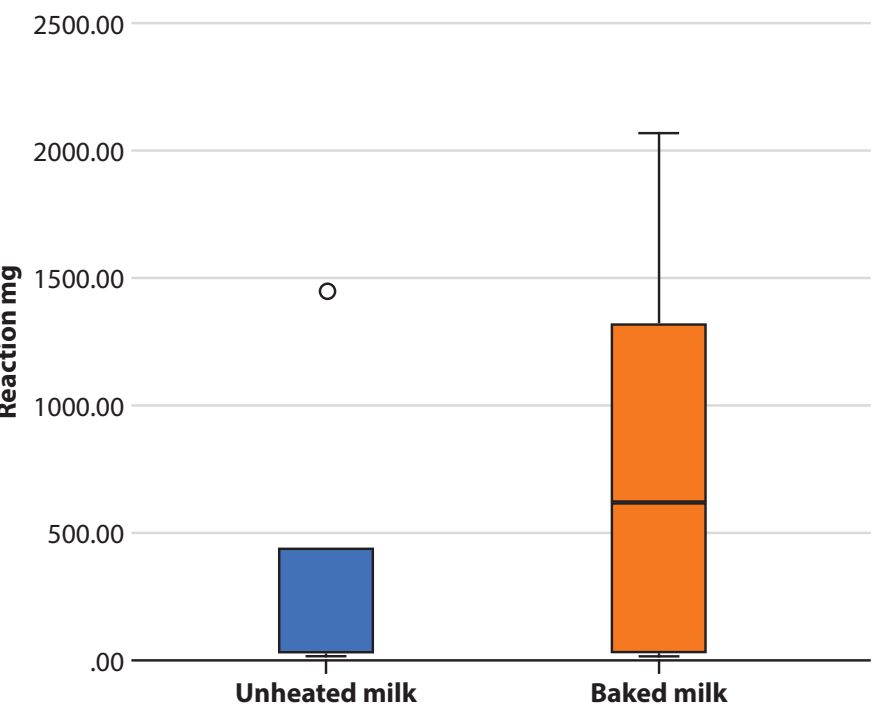

Figure 2. For cases who were reactive to baked milk during OFC, comparison of the amount of baked milk ingested until the development of reaction and the amount of unheated milk ingested until the development of reaction during OFC with unheated milk shows that greater amount of baked milk could be tolerated $(p=0.007)$.

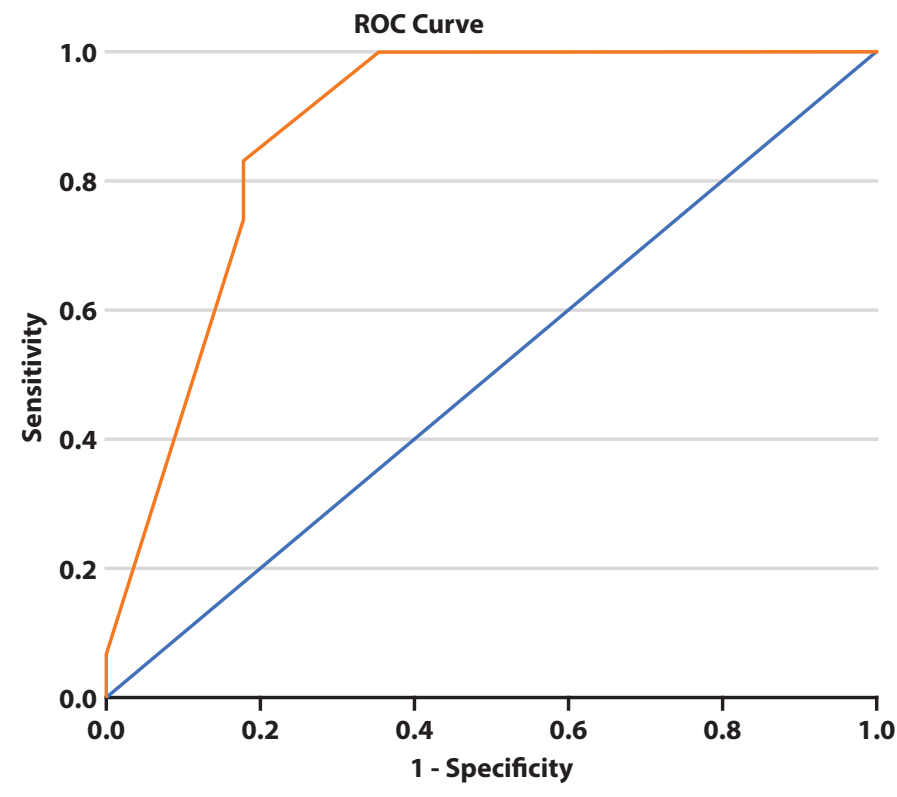

Figure 3. Our ROC curve analysis showed that $620 \mathrm{mg}$ was the optimal cut-off value for the amount of unheated milk ingested during OFC to predict tolerance to baked milk, with a sensitivity of $83.3 \%$ and a specificity of $82.6 \%$ 
until the development of reaction during OFC with unheated milk was significantly greater in the group that could tolerate baked milk $(p<0.05)$ (Table 1).

For cases who could not tolerate baked milk, the amount of baked milk ingested until the development of reaction during OFC was found to be greater than the amount of milk ingested until the development of reaction during OFC with unheated milk $(p<0.05)$ (Figure 2). ROC curve analysis showed that the cut-off value of the amount of milk ingested during OFC with unheated milk for prediction of tolerance to baked milk was $620 \mathrm{mg}$, with $83.3 \%$ sensitivity and $82.6 \%$ specificity. It was area under curve (AUC) $0.88(95 \%$ confidence interval 0.77-0.99). (Figure 3). Additionally, the cut-off value of various tests in predicting clinical reactivity to baked milk was established. $74 \%$ sensitivity and $67 \%$ specificity was established for SPT wheal diameters for baked milk using a cut-off of $3 \mathrm{~mm}, 80 \%$ sensitivity and $70 \%$ specificity was established for SPT wheal diameters for a-lactoalbumin using a cut-off of $4 \mathrm{~mm}, 62 \%$ sensitivity and $70 \%$ specificity was established for a-lactoalbuminspecific IgE using a cut-off of $1.7 \mathrm{kU}_{\mathrm{A}} / \mathrm{L}, 68 \%$ sensitivity and $70 \%$ specificity was established for $\beta$-lactoglobulin-specific IgE using a cut-off of $2.4 \mathrm{kU}_{\mathrm{A}} / \mathrm{L}, 81 \%$ sensitivity and $70 \%$ specificity was established for casein-specific IgE using a cut-off of $0.94 \mathrm{kU}_{\mathrm{A}} / \mathrm{L}$.

\section{Discussion}

For children with cow's milk allergy, the resolution of milk allergy has been shown to occur in shorter time among those who can tolerate baked milk in comparison to those who cannot. ${ }^{3}$ Therefore, it is important to be able to identify children who will tolerate baked milk both for improving the patient's quality of life and accelerating the resolution of allergy. In our study, we found that tolerance to more than $620 \mathrm{mg}$ of milk protein during OFC with unheated milk could predict tolerance to baked milk with $83.3 \%$ sensitivity and $82.6 \%$ specificity. This marker was not previously reported in the literature, and we believe it can be useful in clinical practice.

In their studies, Kwan et al., Barbosa et al., NowakWegrzyn et al. and Cherkaoui et al. evaluated tolerance to baked milk products in cases with cow's milk allergy; they reported that there were no significant differences between cases that were and were not tolerant of baked milk regarding gender, accompanying atopic disease, or familial history of atopy., ${ }^{3,15-17}$ In agreement with the literature, in the present study, we did not find associations between tolerance to baked milk products and gender or accompanying food allergies.

In their study, Nowak-Wegrzyn et al. reported that the median age of onset of milk allergy symptoms was 4 months and found that the age of onset did not show association with baked milk tolerance. ${ }^{3}$ Similarly, the median age of onset of initial symptoms was 5.5 months in our study, and there was no association between age of onset and baked milk tolerance.

Among the studies in which tolerance to baked milk products in cases with cow's milk allergy was evaluated, the median age was 6.6 years in the study by Kim et al. $^{5}$ and 7.3 years in the study by Mehr et al. ${ }^{18}$ Faraj et al., on the other hand, reported a median age of 3.5 years. ${ }^{19}$ In our study, the median age of our cases was 17 months. Similar to these studies, we did not find a significant difference between cases who were and were not tolerant to baked milk products regarding the distribution of age.

In three different studies including children with cow's milk allergy, Bartnikas et al., Kwan et al., and Mehr et al. performed OFC with cakes containing a total of $2.6 \mathrm{~g}$ of milk protein, and the percentages of cases who could tolerate baked milk were reported as $83 \%$ by Bertnikas et al., $60 \%$ by Kwan et al., and $73 \%$ by Mehr et al. ${ }^{4,15,18}$ In contrast to these three studies, Barbosa et al. performed OFC on children with cow's milk allergy with cakes containing a total of $2.8 \mathrm{~g}$ of milk protein, and they reported that $46.7 \%$ of cases could tolerate baked milk. ${ }^{15}$ Unlike other studies, in the present study, we administered a total of $4.5 \mathrm{~g}$ milk protein with a maximum dose of $3 \mathrm{~g}$, which is similar to the amount of milk protein given during OFC with unheated milk. $34.3 \%$ of the cases showed tolerance during OFC with baked milk. The reason why other studies found a higher tolerance rate with baked milk may be that a lower amount of milk protein was given during OFC.

Barbosa et al. reported that total IgE, eosinophil count, milk-specific IgE level, and SPT wheal diameter for milk were not predictive for baked milk tolerance; ${ }^{16}$ and Bartnikas et al. reported that milk-specific IgE or SPT wheal diameter for milk were not associated with baked milk tolerance. ${ }^{4}$ Nowak-Wegrzyn et al. found lower milk-specific IgE level and smaller SPT wheal diameter for milk in the group that tolerated baked milk. ${ }^{3}$ In our study, similar to the literature, we found that milk-specific IgE, SPT wheal diameter for milk, eosinophil count, and total IgE level could not predict tolerance to baked milk.

In their study, Kwan et al. reported that all of the cases who did not develop a reaction to baked milk during SPT could tolerate baked milk during OFC. Additionally, 91\% sensitivity and $61 \%$ specificity was established for SPT wheal diameters for baked milk using a cut-off of $4 \mathrm{~mm}$. They proposed that this could be used as a predictive marker. ${ }^{15} \mathrm{On}$ the contrary, Mehr et al. reported that SPT wheal diameter for baked milk cannot be used as a predictor for tolerance to baked milk. ${ }^{18}$ In our study, the group that tolerated baked milk had smaller SPT wheal diameters. Additionally, of the 14 cases who did not show the reaction to baked milk during SPT, 8 (57\%) could tolerate baked milk. However, SPT wheal diameters for baked milk was a poor predictor for predicting clinical reactivity to baked milk.

Bartnikas et al. reported that $>90 \%$ negative predictive value was established for casein-specific IgE using a cutoff of $0.9 \mathrm{kU}_{\mathrm{A}} / \mathrm{L}$. However, they reported that casein-specific $\operatorname{IgE}$ level was not predictive for baked milk tolerance. ${ }^{4}$ Barbosa et al. reported that both low casein-specific $\operatorname{IgE}$ level and small SPT wheal diameter for milk could predict tolerance to baked milk. ${ }^{16}$ Caubet et al. reported that $74 \%$ Sensitivity and $77 \%$ specificity was established for casein-specific IgE using a cutoff of $4.95 \mathrm{kU}_{\mathrm{A}} / \mathrm{L}^{8}{ }^{8}$ In their studies, Cherkaoui et al. and Nowak-Wegrzyn et al. found lower casein-specific IgE levels in the tolerant group. ${ }^{3,17}$ In agreement with the literature, we found that SPT wheal diameter for casein could not discriminate baked milk tolerance, whereas casein-specific 
IgE level was lower in the group that showed tolerance to baked milk. Casein-specific IgE using a cut-off of $0.94 \mathrm{kU}_{\mathrm{A}} / \mathrm{L}$ could use to predict of baked milk challenge outcome.

Barbosa et al. reported that a-lactoalbumin-specific IgE and $\beta$-lactoglobulin-specific IgE levels, or SPT wheal diameter for $\beta$-lactoglobulin could not discriminate tolerance; however, they found that SPT wheal diameter for a-lactoalbumin was smaller in the tolerant group. ${ }^{16}$ In their studies, Caubet et al. and Nowak-Wegrzyn et al. reported markedly lower $\beta$-lactoglobulin-specific IgE levels in the tolerant group.,8 Similar to the literature, in the present study, we found that both $\beta$-lactoglobulin-specific IgE and $\alpha$-lactoalbumin-specific IgE levels were lower, and SPT wheal diameter for $\alpha$-lactoalbumin was smaller in the tolerant group. However, These markers were poor predictors for predicting clinical reactivity to baked milk.

The main weakness of our study is the lack of sample size. In such types of studies, the comparisons between the variables less powerful and increases the confidence intervals of the results obtained. Nonetheless, we believe that these limitations do not significantly confound the main finding of our study. In addition, we believe our novel marker, which indicates tolerance to baked milk, has limited utility in clinical practice because we think it can only be used to confirm or exclude the diagnosis of milk allergy in patients following OFC test with unheated milk.

\section{Conclusion}

During OFC testing for suspicion of milk allergy, the amount of unheated milk protein that is tolerated by the patient until the development of reaction may be used as a marker to predict tolerance to baked milk. In addition, baked milk products can be given more safely in case milk protein-specific IgE levels are low.

\section{Conflict of interest}

The authors have no conflict of interest to declare

\section{Funding Source}

No external funding was secured for this study.

\section{Financial Disclosure}

The authors have no financial relationships relevant to this article to disclose.

\section{Disclosure statement}

The authors have no conflicts of interest to disclose.

\section{References}

1. Rona RJ, Keil T, Summers C, Gislason D, Zuidmeer L, Sodergren E, et al. The prevalence of food allergy: a meta-analysis. J Allergy Clin Immunol. 2007;120:638-46.

2. Fleisher DM, Perry TT, Atkins D, Wood RA, Burks AW, Jones SM, et al Allergic reactions to foods in preschool-aged children in a prospective observational food allergy study. Pediatrics. 2012;130:25-32.

3. Nowak-Wegrzyn A, Bloom KA, Sicherer SH, Shreffler WG, Noone S, Wanich $\mathrm{N}$, et al. Tolerance to extensively heated milk in children with cow's milk allergy. J Allergy Clin Immunol. 2008;122:342-7.

4. Bartnikas LM, Sheehan WJ, Hoffman EB, Permaul P, Dioun AF Friedlander J, et al. Predicting food challenge outcomes for baked milk: role of specific IgE and skin prick testing. Ann Allergy Asthma Immunol. 2012;109:309-13.

5. Kim JS, Nowak-Wegrzyn A, Sicherer SH, Noone S, Moshier EL, Sampson HA. Dietary baked milk accelerates the resolution of cow's milk allergy in children. J Allergy Clin Immunol. 2011;128:125-31.

6. Goldberg MR, Nachshon L, Appel MY, Elizur A, Levy MB, Eisenberg E, et al. Efficacy of baked milk oral immunotherapy in baked milk-reactive allergic patients. J Allergy Clin Immunol. 2015;136:1601-6.

7. Sato S, Yanagida N, Ogura K, Asaumi T, Okada Y, Koike Y, et al. Immunotherapy in food allergy: towards new strategies. Asian Pac J Allergy Immunol. 2014;23:195-202.

8. Caubet JC, Nowak-Wegrzyn A, Moshier E, Godbold J, Wang J, Sampson HA. Utility of casein-specific IgE levels in predicting reactivity to baked milk. J Allergy Clin Immunol. 2013;131:222-4.

9. Lemon-Mule H, Sampson HA, Sicherer SH, Shreffler WG, Noone S, NowakWegrzyn A. Immunologic changes in children with egg allergy ingesting extensively heated egg. J Allergy Clin Immunol.2008;122: 977-83.

10. Leonard SA. Debates in allergy medicine: baked milk and egg ingestion accelerates resolution of milk and egg allerg. World Allergy Organization J. 2016;9:1.

11. Sampson HA. Utility of food-specific IgE concentrations in predicting symptomatic food allergy. J Allergy Clin Immunol. 2001;107:891-6.

12. Hill DJ, Heine RG, Hosking CS. The diagnostic value of skin prick testing in children with food allergy. Pediatr Allergy Immunol. 2004;15:435-41.

13. Caffarelli C, Ricò S, Rinaldi L, Povesi Dascola C, Terzi C, Bernasconi S. Blood pressure monitoring in children undergoing food challenge: association with anaphylaxis. Ann Allergy Asthma Immunol. 2012;108: 285-6.

14. Muraro A, Halken S, Arsha SH, Beyer K, Dubois AE, Du Toit G, et al. EAACI food allergy and anaphylaxis guidelines. Dragnosis and management of food allergy. Allergy. 2014;69:1008-25.

15. Kwan A, Asper M, Lavi S, Lavine E, Hummel D, Upton JE. Prospective evaluation of testing with baked milk to predict safe ingestion of baked milk in unheated milk-allergic children. Allergy Asthma Clin Immunol. 2016;12:54.

16. Barbosa CPG, Castro APM, Yonamine GH, Gushken AKF, Beck CML, Macedo PRC, et al. Baked milk tolerant patient: Is there any special feature? Allergol Immunopathol. 2017;45:283-9.

17. Cherkaoui S, Bégin P, Paradis L, Paradis J, Des Roches A. Powder milk: a user-friendly and safe product for heated-milk food challenge. Allergy Asthma Clin Immunol. 2015;11:39.

18. Mehr S, Turner PJ, Joshi P, Wong M, Campbell DE. Safety and clinical predictors of reacting to extensively heated cow's milk challenge in cow's milk-allergic children. Ann Allergy Asthma Immunol. 2014;113:425-9.

19. Faraj Z, Kim HL. Skin prick testing with extensively heated milk or egg products helps predict the outcome of an oral food challenge: a retrospective analysis. Allergy Asthma Clin Immunol. 2012;8:5. 